\title{
ON THE RELAXATION OF THE MAXWELL-STEFAN SYSTEM TO LINEAR DIFFUSION
}

\author{
FRANCESCO SALVARANI AND ANA JACINTA SOARES
}

\begin{abstract}
In this note, we rigorously prove the relaxation limit of the Maxwell-Stefan system to a system of heat equations when all binary diffusion coefficients tend to the same positive value.
\end{abstract}

\section{INTRODUCTION}

The Maxwell-Stefan equations have been written in the nineteenth century $[16,18]$, but the interest in their rigorous mathematical study is very recent and not yet complete. After some works, mainly devoted to the matrix formulation of the gradient-flux relationships and described in [10], the study of existence and uniqueness has been carried out in $[2,5,7,14,15,11]$, the formal derivation of multicomponent diffusion equations from the Boltzmann system has been investigated in [4, 6, 3, 12, $13,1]$ and some numerical discretizations of the Maxwell-Stefan system have been proposed in [5, 17].

Despite the advances of the last years, the aforementioned results are far to be complete and many questions are still waiting for a satisfactory answer. Among them, we cite the rigorous study of the relaxation of the Maxwell-Stefan system, under the condition of equimolar diffusion, to a system of heat equations when all binary diffusion coefficients tend to the same positive constant value. In this note, we intend to give a contribution on this last question and fill in the gap in the literature.

\section{THE PROBLEM AND THE MAIN RESULT}

Let $\Omega \subset \mathbb{R}^{d}(d \in \mathbb{N})$ be a bounded domain with regular boundary of class $C^{2}$ and $\varepsilon>0$. Consider a collection of $\varepsilon$-dependent strictly positive and symmetric coefficients $k_{i j}^{\varepsilon}$ (i.e. $k_{i j}^{\varepsilon}=k_{j i}^{\varepsilon}>0$ for all $i, j=1, \ldots, I \in \mathbb{N})$.

The Maxwell-Stefan equations describe a gaseous mixture composed of $I \geq 3$ interacting species. The cross-diffusion relationships link the $\varepsilon$-dependent unknown densities and fluxes $\left(c_{i}^{\varepsilon}, J_{i}^{\varepsilon}\right), i=1, \ldots, I \in$ $\mathbb{N}$, between themselves:

$$
\begin{cases}\partial_{t} c_{i}^{\varepsilon}+\nabla_{x} \cdot J_{i}^{\varepsilon}=0, & (t, x) \in \mathbb{R}^{+} \times \Omega \\ \nabla_{x} c_{i}^{\varepsilon}=-\sum_{j \neq i} k_{i j}^{\varepsilon}\left(c_{j}^{\varepsilon} J_{i}^{\varepsilon}-c_{i}^{\varepsilon} J_{j}^{\varepsilon}\right) . & \end{cases}
$$

Moreover, the fluxes satisfy the equimolar diffusion relationship

$$
\sum_{i=1}^{I} J_{i}^{\varepsilon}=0 .
$$

The $\varepsilon$-dependent coefficients $k_{i j}^{\varepsilon}$ represent the set of binary diffusion coefficients of the gaseous mixture whose behavior is described by the Maxwell-Stefan equations (2.1). Note that the diagonal coefficients $k_{i i}^{\varepsilon}(i=1, \ldots, I)$ play no role in the system.

System (2.1) is supplemented with appropriate initial and boundary conditions. In what follows, we suppose that the solution of (2.1) satisfies homogeneous Neumann boundary conditions, so that we have:

$$
\begin{aligned}
& \left(c_{1}^{\varepsilon}(0, x), \ldots, c_{I}^{\varepsilon}(0, x)\right)=\left(c_{1}^{\text {in }}(x), \ldots, c_{I}^{\text {in }}(x)\right) \in\left(L^{\infty}(\Omega)\right)^{I}, \\
& J_{i}^{\varepsilon}(t, x) \cdot n_{x}=0,(t, x) \in(0, \infty) \times \partial \Omega, i=1, \ldots, I,
\end{aligned}
$$

Key words and phrases. Maxwell-Stefan equations. Cross-diffusion. Relaxation limit.

Work partially funded by the ANR project Kimega (ANR-14-ACHN-0030-01) and by the PHC Pessoa project 7854WM "Derivation of macroscopic PDEs from kinetic theory (mesoscopic scale) and from interacting particle systems (microscopic scale)", Ref. 406/4/4/2017/S. 
where $n_{x} \in \mathbb{S}^{d-1}$ is the outward normal unit vector to the domain $\Omega$ starting from a given point $x \in \partial \Omega$. Since the Maxwell-Stefan system (2.1) is written in terms of molar fractions, we assume that all $c_{i}^{\text {in }} \geq 0$ and

$$
\sum_{i=1}^{I} c_{i}^{\text {in }}(x)=1
$$

Because of the symmetry of the binary diffusion coefficients, it is easy to see, from (2.1) and the equimolar diffusion condition $(2.2)$, that

$$
\sum_{i=1}^{I} c_{i}^{\varepsilon}(t, x)=1
$$

for a.e. $(t, x) \in \mathbb{R}^{+} \times \Omega$. When all the binary diffusion coefficients are equal (i.e. when $k_{i j}^{\varepsilon}=\kappa$ for all $i, j)$, it is easy to see that the Maxwell-Stefan equations (2.1), together with equations (2.2) and (2.4), are equivalent to a system of heat equations for $c_{i}^{\varepsilon}(t, x)$. Indeed, thanks to the equimolar diffusion condition, we have that

$$
J_{i}^{\varepsilon}=-\sum_{j \neq i} J_{j}^{\varepsilon}
$$

and hence, from (2.4) and the second relationship in (2.1), we deduce that

$$
J_{i}^{\varepsilon}=-\frac{1}{\kappa} \nabla_{x} c_{i}^{\varepsilon}
$$

for all $i=1, \ldots, I$, which is the standard form of Fick's law [8,9]. It leads, thanks to the first equation in (2.1), to the system of heat equations

$$
\partial_{t} c_{i}^{\varepsilon}=\frac{1}{\kappa} \Delta_{x} c_{i}^{\varepsilon}, \quad i=1, \ldots, I,
$$

with initial conditions

$$
\left(c_{1}^{\varepsilon}(0, x), \ldots, c_{I}^{\varepsilon}(0, x)\right)=\left(c_{1}^{\mathrm{in}}(x), \ldots, c_{I}^{\mathrm{in}}(x)\right) \in\left(L^{\infty}(\Omega)\right)^{I}
$$

and homogeneous Neumann boundary conditions

$$
\nabla c_{i}^{\varepsilon}(t, x) \cdot n_{x}=0,(t, x) \in(0, \infty) \times \partial \Omega, i=1, \ldots, I .
$$

In this note we prove that, when the binary diffusion coefficients $k_{i j}^{\varepsilon}$ may be different but tend to the same constant value in the limit $\varepsilon \rightarrow 0^{+}$, then the solution of the Maxwell-Stefan system converges to the solution of a suitable system of heat equations. More precisely, our result is the following:

Theorem 2.1. Let $\left(c_{i}^{\varepsilon}, J_{i}^{\varepsilon}\right)$, with $i=1, \ldots, I$ and $I \geq 3$, be the solution of the initial-boundary value problem (2.1)-(2.2)-(2.3), with binary diffusion coefficients of the form

$$
k_{i j}^{\varepsilon}=\kappa+\varepsilon \delta_{i j},
$$

such that $\left|\delta_{i j}\right| \leq 1$ and let $T>0$. Then, in the limit as $\varepsilon \rightarrow 0^{+}$, the densities $\left(c_{1}^{\varepsilon}, \ldots, c_{I}^{\varepsilon}\right)$ converge strongly in $\left(L^{2}((0, T) \times \Omega)\right)^{I}$, with an explicit rate of order $\sqrt{\varepsilon}$, to $\left(c_{1}, \ldots, c_{I}\right)$, and the fluxes $\left(J_{1}^{\varepsilon}, \ldots, J_{I}^{\varepsilon}\right)$ converge strongly in $\left(L^{2}((0, T) \times \Omega)\right)^{d \times I}$ to $\left(J_{1}, \ldots, J_{I}\right)$, with an explicit rate of order $\sqrt{\varepsilon}$.

Moreover, for all $i=1, \ldots, I$, the vector $\left(c_{i}, J_{i}\right)$ solves the set of heat equations

$$
\begin{cases}\partial_{t} c_{i}+\nabla_{x} \cdot J_{i}=0, & (t, x) \in \mathbb{R}^{+} \times \Omega \\ \nabla_{x} c_{i}=-\kappa J_{i}, & \end{cases}
$$

satisfying the initial and boundary conditions

$$
\begin{aligned}
& \left(c_{1}(0, x), \ldots, c_{I}(0, x)\right)=\left(c_{1}^{\text {in }}(x), \ldots, c_{I}^{\text {in }}(x)\right) \in\left(L^{\infty}(\Omega)\right)^{I}, \\
& J_{i} \cdot n_{x}=\nabla c_{i}(t, x) \cdot n_{x}=0,(t, x) \in(0, \infty) \times \partial \Omega, i=1, \ldots, I,
\end{aligned}
$$

i.e. the same as those for the Maxwell-Stefan system. 


\section{THE PROOF}

We base our proof on the following existence and uniqueness result, stated in papers [2, 11]:

Theorem 3.1. Let $\Omega \subset \mathbb{R}^{d}$ be a bounded domain with smooth boundary. Let the initial data $\left(c_{1}^{\text {in }}, \ldots, c_{I}^{\text {in }}\right)$ to the quasi-linear problem (2.1)-(2.2)-(2.3) be non-negative measurable functions such that

$$
\sum_{i=1}^{I} c_{i}^{\text {in }}(x)=1
$$

Then, there exists a unique local-in-time solution - in the $L^{p}$-sense - to the system (2.1)-(2.2)-(2.3) for $t \in(0, T), T>0$. The solution is, in fact, classical.

We now write the flux (gradient) relationship of the Maxwell-Stefan system (2.1) by using both the explicit form of the binary diffusion coefficients given in (2.6) and the equimolar diffusion condition (2.2). We obtain:

$$
\nabla_{x} c_{i}^{\varepsilon}=-\kappa J_{i}^{\varepsilon}-\varepsilon\left(\sum_{j \neq i} \delta_{i j} c_{j}^{\varepsilon}\right) J_{i}^{\varepsilon}+\varepsilon c_{i}^{\varepsilon}\left(\sum_{j \neq i} \delta_{i j} J_{j}^{\varepsilon}\right)
$$

For any vector $v=\left(v_{1}, \ldots, v_{d}\right) \in \mathbb{R}^{d}$, denote by $|v|$ its Euclidean norm, i.e.

$$
|v|=\left(\sum_{l=1}^{d} v_{l}^{2}\right)^{1 / 2}
$$

By performing the scalar product between Equation (3.1) and $J_{i}^{\varepsilon}$ and then by integrating with respect to $x$ in $\Omega$, we deduce

$$
\int_{\Omega} J_{i}^{\varepsilon} \cdot \nabla_{x} c_{i}^{\varepsilon} \mathrm{d} x=-\kappa \int_{\Omega}\left|J_{i}^{\varepsilon}\right|^{2} \mathrm{~d} x-\varepsilon \sum_{j \neq i} \delta_{i j} \int_{\Omega} c_{j}^{\varepsilon}\left|J_{i}^{\varepsilon}\right|^{2} \mathrm{~d} x+\varepsilon \sum_{j \neq i} \delta_{i j} \int_{\Omega} c_{i}^{\varepsilon} J_{j}^{\varepsilon} \cdot J_{i}^{\varepsilon} \mathrm{d} x .
$$

On the other hand, if we multiply the continuity equation in (2.1) by $c_{i}^{\varepsilon}$ and then integrate the resulting equation with respect to $x$ in $\Omega$, we obtain

$$
\frac{1}{2} \frac{\mathrm{d}}{\mathrm{d} t} \int_{\Omega}\left|c_{i}^{\varepsilon}\right|^{2} \mathrm{~d} x=-\int_{\Omega} c_{i}^{\varepsilon} \nabla_{x} \cdot J_{i}^{\varepsilon} \mathrm{d} x=\int_{\Omega} J_{i}^{\varepsilon} \cdot \nabla_{x} c_{i}^{\varepsilon} \mathrm{d} x
$$

where the second equality is a consequence of the homogeneous Neumann boundary conditions for all species.

The previous two equations allow us to conclude that

$$
\frac{1}{2} \frac{\mathrm{d}}{\mathrm{d} t} \int_{\Omega}\left|c_{i}^{\varepsilon}\right|^{2} \mathrm{~d} x=-\kappa \int_{\Omega}\left|J_{i}^{\varepsilon}\right|^{2} \mathrm{~d} x-\varepsilon \sum_{j \neq i} \delta_{i j} \int_{\Omega} c_{j}^{\varepsilon}\left|J_{i}^{\varepsilon}\right|^{2} \mathrm{~d} x+\varepsilon \sum_{j \neq i} \delta_{i j} \int_{\Omega} c_{i}^{\varepsilon} J_{j}^{\varepsilon} \cdot J_{i}^{\varepsilon} \mathrm{d} x .
$$

We know that, for all $i, 0 \leq c_{i}^{\varepsilon} \leq 1$ and, for all $i, j,\left|\delta_{i j}\right| \leq 1$. Hence we can deduce the inequality

$$
\begin{aligned}
\frac{1}{2} \frac{\mathrm{d}}{\mathrm{d} t} \int_{\Omega}\left|c_{i}^{\varepsilon}\right|^{2} \mathrm{~d} x & \leq-\kappa \int_{\Omega}\left|J_{i}^{\varepsilon}\right|^{2} \mathrm{~d} x+\varepsilon(I-1) \int_{\Omega}\left|J_{i}^{\varepsilon}\right|^{2} \mathrm{~d} x+\varepsilon \sum_{j \neq i} \int_{\Omega}\left|J_{j}^{\varepsilon} \cdot J_{i}^{\varepsilon}\right| \mathrm{d} x \\
& \leq-\kappa \int_{\Omega}\left|J_{i}^{\varepsilon}\right|^{2} \mathrm{~d} x+\frac{3}{2} \varepsilon(I-1) \int_{\Omega}\left|J_{i}^{\varepsilon}\right|^{2} \mathrm{~d} x+\frac{\varepsilon}{2} \sum_{j \neq i} \int_{\Omega}\left|J_{j}^{\varepsilon}\right|^{2} \mathrm{~d} x .
\end{aligned}
$$

By summing over all $i$, we have that

$$
\frac{1}{2} \frac{\mathrm{d}}{\mathrm{d} t} \int_{\Omega} \sum_{i=1}^{I}\left|c_{i}^{\varepsilon}\right|^{2} \mathrm{~d} x \leq(-\kappa+2 \varepsilon(I-1)) \int_{\Omega} \sum_{i=1}^{I}\left|J_{i}^{\varepsilon}\right|^{2} \mathrm{~d} x
$$

which implies, for all $\varepsilon<\kappa /(2 I-2)$ and for all $T>0$, that

$$
\int_{\Omega} \sum_{i=1}^{I}\left|c_{i}^{\varepsilon}(t, x)\right|^{2} \mathrm{~d} x \leq \int_{\Omega} \sum_{i=1}^{I}\left|c_{i}^{\mathrm{in}}(x)\right|^{2} \mathrm{~d} x
$$

and, at the same time, that

$$
\int_{(0, T) \times \Omega} \sum_{i=1}^{I}\left|J_{i}^{\varepsilon}\right|^{2} \mathrm{~d} x \mathrm{~d} t \leq \frac{1}{2(\kappa-2 \varepsilon(I-1))} \int_{\Omega} \sum_{i=1}^{I}\left|c_{i}^{\mathrm{in}}(x)\right|^{2} \mathrm{~d} x .
$$


The previous inequality (3.3) proves that, for any $\varepsilon<\kappa /(2 I-2)$, for all $i=1, \ldots, I$ and for all $T>0$, the family $\left\{J_{i}^{\varepsilon}\right\}_{\varepsilon>0}$ is uniformly bounded in $L^{2}((0, T) \times \Omega)$ with respect to $\varepsilon$.

If now we integrate inequality (3.2) with respect to $t$ in $(0, T)$, we deduce that the family $\left\{c_{i}^{\varepsilon}\right\}_{\varepsilon>0}$ is uniformly bounded in $L^{2}((0, T) \times \Omega)$ - with respect to $\varepsilon$ - for any $\varepsilon>0$.

As a consequence of this result, we can deduce a stronger bound for the familiy $\left\{c_{i}^{\varepsilon}\right\}_{\varepsilon>0}$. Indeed, using the elementary inequality

$$
\left(\sum_{k=1}^{n} a_{k}\right)^{2} \leq n \sum_{k=1}^{n} a_{k}^{2}, \quad a_{k} \in \mathbb{R}
$$

from equation (3.1) we have that, for all $\varepsilon<\kappa /(2 I-2)$,

$$
\begin{gathered}
\left|\nabla_{x} c_{i}^{\varepsilon}\right|^{2}=\left|-\left(\varepsilon \sum_{j \neq i} \delta_{i j} c_{j}^{\varepsilon}+\kappa\right) J_{i}^{\varepsilon}+\varepsilon c_{i}^{\varepsilon}\left(\sum_{j \neq i} \delta_{i j} J_{j}^{\varepsilon}\right)\right|^{2} \\
\leq 2\left[(\kappa+\varepsilon)^{2}\left|J_{i}^{\varepsilon}\right|^{2}+\varepsilon^{2}(I-1) \sum_{j \neq i}\left|J_{j}^{\varepsilon}\right|^{2}\right] \leq 2 \kappa^{2}\left[\left(1+\frac{1}{2(I-1)}\right)^{2}\left|J_{i}^{\varepsilon}\right|^{2}+\frac{1}{4(I-1)} \sum_{j \neq i}\left|J_{j}^{\varepsilon}\right|^{2}\right] .
\end{gathered}
$$

By integrating the above inequality with respect to $(t, x)$ in $(0, T) \times \Omega$ and summing over all $i$, we deduce, for all $\varepsilon<\kappa /(2 I-2)$, that

$$
\begin{aligned}
\sum_{i=1}^{I} \int_{(0, T) \times \Omega}\left|\nabla_{x} c_{i}^{\varepsilon}\right|^{2} \mathrm{~d} x \mathrm{~d} t & \leq 2 \kappa^{2}\left[\left(1+\frac{1}{2(I-1)}\right)^{2}+\frac{1}{4}\right] \sum_{i=1}^{I} \int_{(0, T) \times \Omega}\left|J_{i}^{\varepsilon}\right|^{2} \mathrm{~d} x \mathrm{~d} t \\
& \leq 2 \kappa^{2}\left[\left(1+\frac{1}{2(I-1)}\right)^{2}+\frac{1}{4}\right] \frac{1}{2(\kappa-2 \varepsilon(I-1))} \int_{\Omega} \sum_{i=1}^{I}\left|c_{i}^{\mathrm{in}}(x)\right|^{2} \mathrm{~d} x
\end{aligned}
$$

Consider now, for $i=1, \ldots, I \in \mathbb{N}$ the solution $\left(c_{i}, J_{i}\right)$ of the system of heat equations $(2.7)$ with initial and boundary conditions $(2.8)$.

We can prove that $c_{i}^{\varepsilon} \rightarrow c_{i}$ strongly in $L^{2}((0, T) \times \Omega)$ in the limit as $\varepsilon \rightarrow 0^{+}$. Let

$$
g_{i}^{\varepsilon}:=c_{i}^{\varepsilon}-c_{i} \text { and } \Theta_{i}^{\varepsilon}:=J_{i}^{\varepsilon}-J_{i}
$$

for all $i=1 \ldots, I$. Therefore, from systems (2.1) and (2.7), we have that

$$
\left\{\begin{array}{l}
\partial_{t} g_{i}^{\varepsilon}+\nabla_{x} \cdot \Theta_{i}^{\varepsilon}=0, \quad(t, x) \in \mathbb{R}^{+} \times \Omega \\
\nabla_{x} g_{i}^{\varepsilon}=-\kappa \Theta_{i}^{\varepsilon}-\sum_{j \neq i} \varepsilon \delta_{i j}\left(c_{j}^{\varepsilon} J_{i}^{\varepsilon}-c_{i}^{\varepsilon} J_{j}^{\varepsilon}\right),
\end{array}\right.
$$

together with the relationship

$$
\sum_{i=1}^{I} g_{i}^{\varepsilon}=0, \quad \text { for all } t \in \mathbb{R}^{+} \text {and for all } x \in \Omega
$$

with initial and boundary conditions

$$
\begin{aligned}
& \left(g_{1}^{\varepsilon}(0, x), \ldots, g_{I}^{\varepsilon}(0, x)\right)=(0, \ldots, 0) \\
& \Theta_{i}^{\varepsilon}(t, x) \cdot n_{x}=0,(t, x) \in \mathbb{R}^{+} \times \partial \Omega, i=1, \ldots, I .
\end{aligned}
$$

We now multiply the first equation of system (3.5) by $g_{i}^{\varepsilon}$ and then perform the scalar product between $\Theta_{i}$ and both sides of the second equation of system (3.5). After integrating with respect to the spacetime variables in $(0, t) \times \Omega$, with $t \in(0, T)$, and then summing over all $i$, by the fundamental theorem of calculus, and using the initial and boundary conditions $(3.6)$, we deduce, for all $\varepsilon<\kappa /(2 I-2)$, that

$$
\frac{1}{2} \sum_{i=1}^{I} \int_{\Omega}\left[g_{i}^{\varepsilon}(t, x)\right]^{2} \mathrm{~d} x=-\kappa \sum_{i=1}^{I} \int_{(0, t) \times \Omega}\left|\Theta_{i}^{\varepsilon}\right|^{2} \mathrm{~d} x \mathrm{~d} s-\varepsilon \sum_{i=1}^{I} \sum_{j \neq i} \delta_{i j} \int_{(0, t) \times \Omega}\left(c_{j}^{\varepsilon} J_{i}^{\varepsilon}-c_{i}^{\varepsilon} J_{j}^{\varepsilon}\right) \cdot \Theta_{i}^{\varepsilon} \mathrm{d} x \mathrm{~d} s
$$


which implies that

$$
\begin{aligned}
& \frac{1}{2} \sum_{i=1}^{I} \int_{\Omega}\left[g_{i}^{\varepsilon}(t, x)\right]^{2} \mathrm{~d} x \\
& \leq-\kappa \sum_{i=1}^{I} \int_{(0, t) \times \Omega}\left|\Theta_{i}^{\varepsilon}\right|^{2} \mathrm{~d} x \mathrm{~d} s+\varepsilon \sum_{i=1}^{I} \int_{(0, t) \times \Omega}\left(\sum_{j \neq i} c_{j}^{\varepsilon}\right)\left|J_{i}^{\varepsilon} \cdot \Theta_{i}^{\varepsilon}\right| \mathrm{d} x \mathrm{~d} s+\varepsilon \sum_{i=1}^{I} \sum_{j \neq i} \int_{(0, t) \times \Omega} c_{i}^{\varepsilon}\left|J_{j}^{\varepsilon} \cdot \Theta_{i}^{\varepsilon}\right| \mathrm{d} x \mathrm{~d} s
\end{aligned}
$$

where we used the bound $\left|\delta_{i j}\right| \leq 1$. Thanks to the elementary inequality $2|a \cdot b| \leq|a|^{2}+|b|^{2}$ and to condition (2.4), we obtain

$$
\frac{1}{2} \sum_{i=1}^{I} \int_{\Omega}\left[g_{i}^{\varepsilon}(t, x)\right]^{2} \mathrm{~d} x \leq-\left(\kappa-\frac{\varepsilon}{2} I\right) \sum_{i=1}^{I} \int_{(0, t) \times \Omega}\left|\Theta_{i}^{\varepsilon}\right|^{2} \mathrm{~d} x \mathrm{~d} s+\frac{\varepsilon}{2} I \sum_{i=1}^{I} \int_{(0, t) \times \Omega}\left|J_{i}^{\varepsilon}\right|^{2} \mathrm{~d} x \mathrm{~d} s .
$$

We finally deduce

$$
\frac{1}{2} \sum_{i=1}^{I} \int_{\Omega}\left[g_{i}^{\varepsilon}(t, x)\right]^{2} \mathrm{~d} x \leq \frac{\varepsilon}{2} I \sum_{i=1}^{I} \int_{(0, T) \times \Omega}\left|J_{i}^{\varepsilon}\right|^{2} \mathrm{~d} x \mathrm{~d} s \leq \frac{\varepsilon I T}{4(\kappa-2 \varepsilon(I-1))} \sum_{i=1}^{I} \int_{\Omega}\left|c_{i}^{\text {in }}(x)\right|^{2} \mathrm{~d} x,
$$

where the last inequality is a direct consequence of estimate (3.3). Since the last expression in the above inequality is independent of $t$, we can hence conclude that

$$
\sum_{i=1}^{I}\left\|g_{i}^{\varepsilon}\right\|_{L^{2}((0, T) \times \Omega)}^{2}=\sum_{i=1}^{I}\left\|c_{i}^{\varepsilon}-c_{i}\right\|_{L^{2}((0, T) \times \Omega)}^{2} \leq \frac{\varepsilon I T}{2(\kappa-2 \varepsilon(I-1))} \sum_{i=1}^{I} \int_{\Omega}\left|c_{i}^{\mathrm{in}}(x)\right|^{2} \mathrm{~d} x .
$$

We have hence that

$$
c_{i}^{\varepsilon} \rightarrow c_{i} \text { strongly in } L^{2}((0, T) \times \Omega) \text { as } \varepsilon \rightarrow 0^{+}
$$

with a rate of order at least $\sqrt{\varepsilon}$.

Moreover, from (3.7) and estimate (3.3), we deduce that

$$
\left(\kappa-\frac{\varepsilon}{2} I\right) \sum_{i=1}^{I} \int_{(0, t) \times \Omega}\left|\Theta_{i}^{\varepsilon}\right|^{2} \mathrm{~d} x \mathrm{~d} s \leq \frac{\varepsilon}{2} I \sum_{i=1}^{I} \int_{(0, t) \times \Omega}\left|J_{i}^{\varepsilon}\right|^{2} \mathrm{~d} x \mathrm{~d} s \leq \frac{\varepsilon I T}{4(\kappa-2 \varepsilon(I-1))} \sum_{i=1}^{I} \int_{\Omega}\left|c_{i}^{\text {in }}(x)\right|^{2} \mathrm{~d} x .
$$

The previous inequality allows to conclude that

$$
\sum_{i=1}^{I}\left\|\Theta_{i}^{\varepsilon}\right\|_{\left(L^{2}((0, T) \times \Omega)\right)^{d}}^{2}=\sum_{i=1}^{I}\left\|J_{i}^{\varepsilon}-J_{i}\right\|_{\left(L^{2}((0, T) \times \Omega)\right)^{d}}^{2} \leq \frac{\varepsilon I T}{2(\kappa-2 \varepsilon(I-1))(2 \kappa-\varepsilon I)} \sum_{i=1}^{I} \int_{\Omega}\left|c_{i}^{\mathrm{in}}(x)\right|^{2} \mathrm{~d} x .
$$

We finally have that

$$
J_{i}^{\varepsilon} \rightarrow J_{i} \text { strongly in }\left(L^{2}((0, T) \times \Omega)\right)^{d \times I} \text { as } \varepsilon \rightarrow 0^{+}
$$

with a rate of order at least $\sqrt{\varepsilon}$.

Consider now Equation (3.1). Because of the $L^{2}$ bounds on $c_{i}^{\varepsilon}$ and $J_{i}^{\varepsilon}$ given by (3.2) and (3.3), we deduce that

$$
-\varepsilon\left(\sum_{j \neq i} \delta_{i j} c_{j}^{\varepsilon}\right) J_{i}^{\varepsilon}+\varepsilon c_{i}^{\varepsilon}\left(\sum_{j \neq i} \delta_{i j} J_{j}^{\varepsilon}\right) \rightarrow 0
$$

weakly in $L^{2}((0, T) \times \Omega)^{d}$. As a consequence, the limits $c_{i}$ and $J_{i}$ satisfy the heat equation system (2.7) in distributional sense with homogeneous Neumann boundary conditions.

\section{REFERENCES}

[1] B. Anwasia, P. Gonçalves, and A. J. Soares. From the simple reacting sphere kinetic model to the reaction-diffusion system of Maxwell-Stefan type. preprint arXiv:170\%.01316, 2017.

[2] D. Bothe. On the Maxwell-Stefan approach to multicomponent diffusion. In Parabolic problems, volume 80 of Progr. Nonlinear Differential Equations Appl., pages 81-93. Birkhäuser/Springer Basel AG, Basel, 2011.

[3] L. Boudin, B. Grec, and V. Pavan. The Maxwell-Stefan diffusion limit for a kinetic model of mixtures with general cross sections. Nonlinear Analysis, 159:40-61, 2017.

[4] L. Boudin, B. Grec, M. Pavić, and F. Salvarani. Diffusion asymptotics of a kinetic model for gaseous mixtures. Kinet. Relat. Models, 6(1):137-157, 2013.

[5] L. Boudin, B. Grec, and F. Salvarani. A mathematical and numerical analysis of the Maxwell-Stefan diffusion equations. Discrete Contin. Dyn. Syst. Ser. B, 17(5):1427-1440, 2012.

[6] L. Boudin, B. Grec, and F. Salvarani. The Maxwell-Stefan diffusion limit for a kinetic model of mixtures. Acta Appl. Math., 136:79-90, 2015. 
[7] X. Chen and A. Jüngel. Analysis of an Incompressible Navier-Stokes-Maxwell-Stefan System. Comm. Math. Phys., 340(2):471-497, 2015.

[8] A. Fick. On liquid diffusion. Phil. Mag., 10(63):30-39, 1855.

[9] A. Fick. Über Diffusion. Poggendorff's Annel Physik, 94:59-86, 1855.

[10] V. Giovangigli. Multicomponent flow modeling. Modeling and Simulation in Science, Engineering and Technology. Birkhäuser Boston Inc., Boston, MA, 1999.

[11] M. Herberg, M. Meyries, J. Prüss, and M. Wilke. Reaction-diffusion systems of Maxwell-Stefan type with reversible mass-action kinetics. Nonlinear Analysis, 159:264-284, 2017.

[12] H. Hutridurga and F. Salvarani. Maxwell-Stefan diffusion asymptotics for gas mixtures in non-isothermal setting. Nonlinear Analysis, 159:285-297, 2017.

[13] H. Hutridurga and F. Salvarani. On the Maxwell-Stefan diffusion limit for a mixture of monatomic gases. Math. Methods Appl. Sci., 40(3):803-813, 2017.

[14] H. Hutridurga and F. Salvarani. Existence and uniqueness analysis of a non-isothermal cross-diffusion system of Maxwell-Stefan type. Appl. Math. Letters, 75:108-113, 2018.

[15] A. Jüngel and I. V. Stelzer. Existence analysis of Maxwell-Stefan systems for multicomponent mixtures. SIAM J. Math. Anal., 45(4):2421-2440, 2013.

[16] J. C. Maxwell. On the dynamical theory of gases. Phil. Trans. R. Soc., 157:49-88, 1866.

[17] M. McLeod and Y. Bourgault. Mixed finite element methods for addressing multi-species diffusion using the MaxwellStefan equations. Comput. Methods Appl. Mech. Engrg., 279:515-535, 2014.

[18] J. Stefan. Über das Gleichgewicht und die Bewegung insbesondere die Diffusion von Gasgemengen. Akad. Wiss. Wien, 63:63-124, 1871.

F.S.: Université Paris-Dauphine, PSL Research University, Ceremade, UMR CNRS 7534, F-75775 Paris Cedex 16, France \& Università degli Studi di Pavia, Dipartimento di Matematica, I-27100 Pavia, Italy

E-mail address: francesco.salvarani@unipv.it

A.J.S.: University of Minho, Centre of Mathematics, 4710-057 Braga, Portugal

E-mail address: ajsoares@math.uminho.pt 